IASSNS-HEP-00/33

SU-ITP 00-13

hep-th/0005040

\title{
Strings in Background Electric Field, Space/Time Noncommutativity and A New Noncritical String Theory
}

\author{
N. Seiberg, \\ School of Natural Sciences \\ Institute for Advanced Study \\ Olden Lane, Princeton, NJ 08540 \\ L. Susskind and N. Toumbas \\ Department of Physics \\ Stanford University \\ Stanford, CA 94305-4060
}

Searching for space/time noncommutativity we reconsider open strings in a constant background electric field. The main difference between this situation and its magnetic counterpart is that here there is a critical electric field beyond which the theory does not make sense. We show that this critical field prevents us from finding a limit in which the theory becomes a field theory on a noncommutative spacetime. However, an appropriate limit toward the critical field leads to a novel noncritical string theory on a noncommutative spacetime.

$5 / 00$ 


\section{Introduction}

The recent interest in noncommutative geometry and its appearance in string theory [1]3], has been limited almost exclusively to the case where the noncommutative coordinates are spacelike:

$$
\left[x^{i}, x^{j}\right]=i \theta^{i j} .
$$

The time direction, $x^{0}$, remained unaffected and the commutator

$$
\left[x^{0}, x^{i}\right]=i \theta^{0 i}
$$

was set to zero.

It is interesting to ask what happens, if $\theta^{0 i} \neq 0$. The meaning of (1.2) in this case is not completely clear. In quantum mechanics the time coordinate labels the evolution of the system. Unlike the coordinates of particles, the time is not an operator, and hence, it is not clear how it could fail to commute. Nevertheless, there are several motivations for studying such a situation:

1. Equation (1.2) with nonzero $\theta^{0 i}$ is a natural extension of (1.1), and as such it appears like an interesting situation to explore.

2. Noncommutativity in space (1.1) occurs when D-branes are placed in a nonzero $B$ field with components along the space-space directions. Equivalently, it occurs when the D-branes have background magnetic field. Similarly, $\theta^{0 i}$ can be activated by turning on $B_{0 i}$ or by placing the D-branes in background electric field. Therefore, these are physical situations which can occur and as such should be understood.

3. The commutator (1.2) leads to an uncertainty relation between time and space of the form

$$
\Delta x^{0} \Delta x^{i} \neq 0 .
$$

Such an uncertainty relation was advocated as a generic property of string theory "the stringy uncertainty principle" even when no electric field is present (see [⿴囗⿰丿㇄口阝 for a review and an extensive list of earlier references). In [5] we explore some of the consequences of such an uncertainty principle.

4. The stringy uncertainty principle (1.3) and the space/time commutator (1.2) play a crucial role in the proposal of [6] regarding the information puzzle in black-holes.

5. As we said above, lack of commutativity of time appears to be in serious conflict with our current understanding of quantum mechanics. Better understanding of this issue can perhaps shed light on the role of time in string theory. 
Gauge transformation on a noncommutative space are different than on a commutative space. However, as in [3], there exists a change of variables from noncommutative to commutative gauge fields allowing us to express the effective Lagrangian in terms of ordinary commutative gauge fields. This transformation applies to the space/time as well as to the space/space noncommutativity and it resolves some of the mysteries associated with such gauge fields. The effective Lagrangian after the transformation still includes an infinite number of space and time derivatives and questions of unitarity and causality might still be present. However, in the specific example of string theory in background electric field the first quantized description makes it clear that, at least in perturbation theory, the theory is unitary and causal.

Motivated by these considerations we will study strings in background electric field. Our main concern will be the question of whether there exists a limit in which the full string theory simplifies. One's first hope is that as for magnetic backgrounds [3], there exists an $\alpha^{\prime} \rightarrow 0$ limit in which the theory becomes field theoretic and hence more tractable. One of our conclusions is that this is not the case. The best we could find is a limit of the theory in which the closed strings including the gravitational degrees of freedom decouple and we are left with a simpler theory of open strings only. However, unlike the magnetic case, this theory is not a field theory but a string theory.

\section{The setup}

We will be studying D-branes in flat $\mathcal{R}^{n}$ with metric $g_{\mu \nu}$ and constant $B$ field $B_{\mu \nu}$. We can pick a frame in which $g_{\mu \nu}=\eta_{\mu \nu}$, but since we are planning to scale the metric we do not do that. For simplicity we will take the metric $g_{\mu \nu}$ to be diagonal. Then it is easy to distinguish between the spatial and the temporal directions.

The boundary conditions for open strings are

$$
\left.\left(g_{\mu \nu} \partial_{\sigma} x^{\nu}+2 \pi \alpha^{\prime} B_{\mu \nu} \partial_{\tau} x^{\nu}\right)\right|_{\text {boundary }}=0
$$

where $\tau$ is tangent to the boundary and $\sigma$ is normal to the boundary. The analysis of [3], which was motivated by [7], leads to the definition of the open string metric $G_{\mu \nu}$, the noncommutativity parameter $\theta^{\mu \nu}$ and the open string coupling constant $G_{s}$

$$
\begin{aligned}
& G_{\mu \nu}=g_{\mu \nu}-\left(2 \pi \alpha^{\prime}\right)^{2}\left(B g^{-1} B\right)_{\mu \nu} \\
& \theta^{\mu \nu}=2 \pi \alpha^{\prime}\left(\frac{1}{g+2 \pi \alpha^{\prime} B}\right)_{A}^{\mu \nu} \\
& G_{s}=g_{s}\left(\frac{\operatorname{det} G_{\mu \nu}}{\operatorname{det}\left(g_{\mu \nu}+2 \pi \alpha^{\prime} B_{\mu \nu}\right)}\right)^{\frac{1}{2}}
\end{aligned}
$$


where ()$_{A}$ denotes the antisymmetric part and $g_{s}$ is the closed string coupling.

As is well known the backgound $B_{\mu \nu}$ can be traded for background $F_{\mu \nu}$ on the branes. Most of the analysis in the literature is devoted to the magnetic case, where only the spatial components of $B$ (or $F$ ) are nonzero. Here we will be interested in the purely electric case with $B_{i j}=F_{i j}=0$, but $B_{0 i} \neq 0$. The generalization to the case of both electric and magnetic background fields is straightforward.

Without loss of generality we can choose a frame in which the electric field points in the $x^{1}$ direction. Focusing on $x^{0,1}$ we take

$$
g_{\mu \nu}=g\left(\begin{array}{cc}
-1 & 0 \\
0 & 1
\end{array}\right), \quad B_{\mu \nu}=\left(\begin{array}{cc}
0 & E \\
-E & 0
\end{array}\right) .
$$

We also find it convenient to define the dimensionless electric field

$$
\widetilde{E}=\frac{2 \pi \alpha^{\prime} E}{g}=\frac{E}{E_{c r}}
$$

where $E_{c r}=\frac{g}{2 \pi \alpha^{\prime}}$ is the critical electric field. In this frame the open string parameters (1.5) become

$$
\begin{aligned}
& G_{\mu \nu}=g_{\mu \nu}-\left(2 \pi \alpha^{\prime}\right)^{2}\left(B g^{-1} B\right)_{\mu \nu}=\frac{g^{2}-\left(2 \pi \alpha^{\prime} E\right)^{2}}{g}\left(\begin{array}{cc}
-1 & 0 \\
0 & 1
\end{array}\right)_{\mu \nu} \equiv G\left(\begin{array}{cc}
-1 & 0 \\
0 & 1
\end{array}\right)_{\mu \nu} \\
& \theta^{\mu \nu}=\frac{\left(2 \pi \alpha^{\prime}\right)^{2} E}{g^{2}-\left(2 \pi \alpha^{\prime} E\right)^{2}}\left(\begin{array}{cc}
0 & 1 \\
-1 & 0
\end{array}\right)^{\mu \nu} \equiv \theta\left(\begin{array}{cc}
0 & 1 \\
-1 & 0
\end{array}\right)^{\mu \nu} \\
& G_{s}=g_{s}\left(\frac{\operatorname{det} G_{\mu \nu}}{\operatorname{det}\left(g_{\mu \nu}+2 \pi \alpha^{\prime} B_{\mu \nu}\right)}\right)^{\frac{1}{2}}=g_{s}\left(1-\left(\frac{2 \pi \alpha^{\prime} E}{g}\right)^{2}\right)^{\frac{1}{2}} .
\end{aligned}
$$

The constants $G$ and $\theta$ can be expressed as

$$
\begin{aligned}
& G=g\left(1-\widetilde{E}^{2}\right) \\
& \theta=\frac{1}{E_{c r}} \frac{\widetilde{E}}{1-\widetilde{E}^{2}} \\
& G_{s}=g_{s}\left(1-\widetilde{E}^{2}\right)^{\frac{1}{2}} .
\end{aligned}
$$

In the case of $N$ branes the effective 't Hooft coupling constant is

$$
G_{e f f}=N G_{s}=N g_{s}\left(1-\widetilde{E}^{2}\right)^{\frac{1}{2}}
$$

In section 2 we study strings in background electric field. We first review and extend the canonical quantization of the first quantized theory on the strip, and then discuss the 
interactions. We end with a few comments about the phenomenon of critical electric field. In section 3 we show that there is no zero slope limit in which the theory becomes a field theory on a noncommutative spacetime. Our novel noncritical string theory on such a spacetime is presented in section 4 .

As we were completing this work we learned of a closely related work by R. Gopakumar, J. Maldacena, S. Minwalla and A. Strominger [8].

\section{Strings In Background Electric Field}

\subsection{Canonical quantization on the strip}

In this subsection we follow [9.10] and analyze the spectrum of the first quantized open string in the background of constant electric field.

In terms of the spacetime lightcone coordinates $x^{ \pm}=\frac{1}{\sqrt{2}}\left(x^{0} \pm x^{1}\right)$ the boundary conditions (1.4) become

$$
\left.\left(\partial_{\sigma} x^{ \pm} \mp \widetilde{E} \partial_{\tau} x^{ \pm}\right)\right|_{\sigma=0, \pi}=0 .
$$

The solution of the worldsheet equations of motion subject to these boundary conditions are

$$
\begin{aligned}
x^{ \pm}(\sigma, \tau)= & x_{0}^{ \pm}+\alpha^{\prime} p^{ \pm}(\tau \pm \widetilde{E} \sigma)-i\left(\alpha^{\prime}\right)^{\frac{1}{2}} \sum_{n \neq 0} \frac{a_{n}^{ \pm}}{n}\left[(1 \pm \widetilde{E}) e^{i n(\sigma+\tau)}+(1 \mp \widetilde{E}) e^{i n(-\sigma+\tau)}\right] \\
= & x_{0}^{ \pm}+\frac{1}{2} \alpha^{\prime} p^{ \pm}[(\tau+\sigma)(1 \pm \widetilde{E})+(\tau-\sigma)(1 \mp \widetilde{E})] \\
& \quad-i\left(\alpha^{\prime}\right)^{\frac{1}{2}} \sum_{n \neq 0} \frac{a_{n}^{ \pm}}{n}\left[(1 \pm \widetilde{E}) e^{i n(\sigma+\tau)}+(1 \mp \widetilde{E}) e^{i n(-\sigma+\tau)}\right],
\end{aligned}
$$

with reality conditions $\left(a_{n}^{ \pm}\right)^{*}=a_{-n}^{ \pm}$.

We note that as $E$ approaches the critical field $\pm E_{c r}(\widetilde{E} \rightarrow \pm 1)$, the modes of $x^{ \pm}$ become chiral in the worldsheet sense.

As in [10], the commutation relations of the modes in (2.2) are

$$
\begin{aligned}
& {\left[x_{0}^{\mu}, x_{0}^{\nu}\right]=i \theta^{\mu \nu},} \\
& {\left[x_{0}^{\mu}, p^{\nu}\right]=i 2 G^{\mu \nu},} \\
& {\left[a_{n}^{\mu}, a_{m}^{\nu}\right]=\frac{1}{2} n \delta_{n+m, 0} G^{\mu \nu},}
\end{aligned}
$$


and the worldsheet Hamiltonian is given by

$$
L_{0}=\frac{1}{4} \alpha^{\prime} G_{\mu \nu} p^{\mu} p^{\nu}+2 G_{\mu \nu} \sum_{n=1}^{\infty} a_{-n}^{\mu} a_{n}^{\nu}+L_{0}^{t r},
$$

where $L_{0}^{t r}$ is the contribution of the transverse directions. This Hamiltonian determines the masses of the various string modes. It is important that the relevant metric in all these expressions is the open string metric $G_{\mu \nu}$ of (1.5) (1.8).

In order to gain some intuition to the physics of the problem we neglect the oscillators in $(2.2)$

$$
\bar{x}^{ \pm}(\sigma, \tau)=x_{0}^{ \pm}+\alpha^{\prime} p^{ \pm}(\tau \pm \widetilde{E} \sigma)
$$

As in [11]13] (see also [3]), in this approximation the string is a rod which behaves like a dipole - the two ends of the string carry opposite charges. Two important properties of $\bar{x}$ are the following

1. It is easy to check using (2.3) that its center of mass coordinates, $\widetilde{x}_{0}^{ \pm}=x_{0}^{ \pm} \pm \frac{\pi}{2} \alpha^{\prime} p^{ \pm} \widetilde{E}$, commute. The lack of commutativity of $x_{0}^{\mu}$ in (2.3) occurs only because these are the boundaries of the string.

2. The length of the rod is proportional to Ep. As in the magnetic case, the size of the dipole is proportional to the background field and the momentum [11-13]. However, here, the dipole grows in the longitudial direction rather than in the transverse direction. This fact plays a crucial role in [5].

In order to find the physical spectrum of the string we should remember to include the ghosts and look for the BRST cohomology. Instead, we can try to use a physical gauge like a lightcone gauge. The boundary conditions (2.1) do not allow us to choose $x^{+}=\alpha^{\prime} p^{+} \tau$. Instead, a natural guess for the lightcone gauge in this system is

$$
x^{+}=\alpha^{\prime} p^{+}(\tau+\widetilde{E} \sigma)
$$

The choice (2.6) is natural because it satisfies the equation of motion and the boundary conditions. In order to prove it, we replace the previous choice of the conformal gauge with the metric $\left(\begin{array}{ll}-1 & \\ & 1\end{array}\right)$ by a gauge choice with the worldsheet metric $\left(\begin{array}{cc}-1 & \widetilde{E} \\ \widetilde{E} & 1-\widetilde{E}^{2}\end{array}\right)$. Then we can choose the standard lightcone gauge $x^{+}=\alpha^{\prime} p^{+} \tau$. Finally, we change the worldsheet coordinates to a frame with a metric $\left(\begin{array}{cc}-1 & \\ & 1\end{array}\right)$ to find (2.6).

In the lightcone gauge (2.6) the oscillators in $a^{ \pm}$are absent and the physical spectrum of the theory is essentially that of the transverse directions without the electric field. The 
only effect of the electric field is to change the metric $g_{\mu \nu}$ to the open string metric $G_{\mu \nu}$ as in (1.5). This metric appears in the mass shell condition through the Hamiltonian (2.4).

Examining (2.6) (or (2.5)) we see that for fixed $\tau$ the two ends of the string are not at the same value of $x^{+}$. The two ends of the string are always spacelike separated. As the electric field becomes critical $\widetilde{E}= \pm 1$ the two end points at fixed $\tau$ become lightlike separated. This gives another perspective to the impossibility of having larger values of the electric field.

This picture leads to another interpretation to the fuzziness in $x^{+}$corresponding to the noncommutativity in it. This is important because in the lightcone gauge $x^{+}$is treated as a parameter, labeling the evolution of the system and therefore there is superficially no room for any fuzziness in it. The fuzziness occurs because the string is not point-like but is like a rod whose two end points are at different times. This is similar to the origin of the fuzziness of space in the magnetic case [12] which originates from the size of the dipole.

An important difference between the electric and the magnetic backgrounds is the following. For magnetic backgrounds the dipole grows in the direction transverse to the motion by amount proportional to $B_{i j} p^{i}$, while for electric backgrounds the growth is in the longitudinal direction by amount proportional to $B_{0 i} p^{i}$.

Finally we would like to resolve a little puzzle. The discussion of the noncommutative field theory in 12 derives the size of the dipole as $\Delta x^{\mu}=\theta^{\mu \nu} p_{\nu}$, while from the normal mode expansion of the zero mode, as in (2.5), the size is $\Delta x^{\mu}=-\left(2 \pi \alpha^{\prime}\right)^{2} g^{\mu \nu} B_{\nu \rho} p^{\rho}$. How can these two expressions be the same? The answer is that in the noncommutative field theory we use the open string metric $G_{\mu \nu}$ to raise and lower indices. Using the identity $\theta^{\mu \nu} G_{\nu \rho}=-\left(2 \pi \alpha^{\prime}\right)^{2} g^{\mu \nu} B_{\nu \rho}$ (which follows from (1.5)) and $p_{\alpha}=G_{\alpha \beta} p^{\beta}$ it is easy to show that these two expressions for the size of the dipole are identical.

\subsection{String interactions}

The discussion so far was limited to the free first quantized theory and its spectrum on the strip. In order to include interactions using vertex operators we must use a covariant gauge. Otherwise we cannot change the value of $p^{+}$, and we cannot probe the noncommutative phases $p_{1}^{+} p_{2}^{-} \theta$ which are characteristic of the noncommutative theory.

At the level of the disc amplitudes the inclusion of the electric field is straightforward. As explained in [3], all we need to do is to start with the corresponding amplitudes for $E=0$

and replace $g_{\mu \nu}$ by $G_{\mu \nu}, g_{s}$ by $G_{s}$ and multiply the answer by the standard phase factor with the noncommutativity parameter $\theta$. The fact that $g$ is replaced with $G$ is consistent with 
the replacement of $g^{\mu \nu} p_{\mu} p_{\nu}$ by $G^{\mu \nu} p_{\mu} p_{\nu}$ in the mass formula (the worldsheet Hamiltonian (2.4)). These observations are consistent with the explicit calculations performed in the special case of scattering on D1-branes in [14].

\subsection{The critical electric field}

At $E=E_{c r}$ the various expressions for the open string parameters (1.9) are singular. The origin of this singularity was understood in [9]. An open string should be thought of as a dipole whose ends carry opposite charges. When an open string is stretched along the $x^{1}$ direction in the orientation preferred by the background electric field, the field reduces its energy. For $|E| \lesssim E_{c r}$, the energy stored in the tension of the string is almost balanced by the electric energy of the stretched string. More quantitatively, the effective tension of such a string is

$$
\frac{1}{2 \pi \alpha_{\text {eff }}^{\prime}}=\frac{1}{2 \pi \alpha^{\prime}} \frac{G_{11}}{g}=\frac{1-\widetilde{E}^{2}}{2 \pi \alpha^{\prime}},
$$

and it vanishes as $|E| \rightarrow E_{c r}$. Beyond that value virtual strings can materialize out of the vacuum, stretch to infinity and destabilize the vacuum.

When the string is streched along different directions this effect is absent and its tension is not reduced. Instead of thinking of a varied tension, it is easier to keep the tension fixed at $\frac{1}{2 \pi \alpha^{\prime}}$ and scale the length. Equivalently, we scale the metric. This is the intuitive reason for the expression for the open string metric $G_{\mu \nu}$ which has the effect of reducing the metric in the directions of $x^{0}$ and $x^{1}$ but keeping $G_{\mu \nu}=g_{\mu \nu}$ in the other directions.

The expression for the effective open string coupling constant (1.9)

$$
G_{s}=g_{s}\left(1-\widetilde{E}^{2}\right)^{\frac{1}{2}}
$$

is also interesting. We note that it vanishes as $E$ approaches the critical field for fixed value of the underlying string coupling constant $g_{s}$. This fact has a simple reason. Consider the transition of one of the light open strings to a closed string. For this process to take place, the stretched open string needs to bend over in order for its ends to touch each other. Then, part of it will be stretched against the electric field and will be very heavy. Therefore, such a transition cannot happen easily and correspondingly the effective coupling constant is small.

Finally, let us compactify $x^{1}$ and use T-duality along the compact direction. We get branes of one lower dimension moving with velocity $v=\widetilde{E}$ around the circle. As the 
electric field approaches its critical value, the velocity of the branes approaches the speed of light and the momentum of the branes becomes large. We can then make a large boost to bring the branes to rest. In the T-dual picture of the original theory, this amounts to boosting to large momentum. Therefore, studying the electric theory near the critical limit is equivalent to studying the theory in the DLCQ description.

Below we will study the limit $E \rightarrow E_{c r}$ in more detail. We will define an appropriate scaling limit which focuses on these light strings and find an effective theory of these modes only.

\section{Looking For A Zero Slope Limit With Noncommutative Spacetime}

In this section we will look for a field theory limit of string theory in electric field background, which has a noncommutative spacetime. Naively, all we have to do is repeat the analysis of [3] to find a zero slope limit. However, we will show that this is not possible.

In our discussion we will hold the coordinates and external momenta fixed, and will rescale the metric and $\alpha^{\prime}$. An alternate description in terms of fixed metric and $\alpha^{\prime}$ but rescaled coordinates and momenta is, of course, also possible.

We start by reviewing the propagator along the boundary of the disc

$$
\left\langle x^{\mu}(\tau) x^{\nu}(0)\right\rangle=-\alpha^{\prime} G^{\mu \nu} \log \tau^{2}+i \frac{\theta^{\mu \nu}}{2} \epsilon(\tau)
$$

where the coefficients of the two terms in the propagator are given in terms of

$$
\begin{aligned}
& \theta=\frac{E}{E_{c r}^{2}-E^{2}}=\frac{1}{E_{c r}} \frac{\widetilde{E}}{1-\widetilde{E}^{2}} \\
& \alpha^{\prime} G^{-1}=\frac{1}{2 \pi E_{c r}\left(1-\widetilde{E}^{2}\right)}=\frac{1}{2 \pi} \frac{\theta}{\widetilde{E}} .
\end{aligned}
$$

We are looking for a limit subject to the following requirements:

1. We want $\alpha^{\prime} \rightarrow 0$, so that all the oscillator modes decouple and the resulting theory is field theoretic. Otherwise, the theory has an infinite number of massive string modes.

2. We should keep $G$ finite. The reason for that is that $G_{\mu \nu}$ will be the metric of the resulting theory, which should be finite.

3. The noncommutativity parameter $\theta$ should be finite, since we want the resulting theory to be noncommutative. 
Since $|\widetilde{E}|<1$, if $\theta$ is kept fixed, $\alpha^{\prime} G^{-1}$ cannot be taken to zero. Therefore, the first term in the propagator (3.1) cannot be neglected and the directions $x^{0,1}$ remain stringy. This is unlike the situation for magnetic backgrounds, where there is no bound on the background magnetic field, and it is possible to take the analog of $\widetilde{E}$ to infinity and remove the first term in the propagator, thus making the theory in these directions field theoretic [3].

Roughly, for magnetic backgrounds the scaling is such that the magnetic field goes to infinity. Here, we cannot scale $E$ to infinity, and therefore we cannot reach a zero slope limit.

One might argue that perhaps we should scale $\alpha^{\prime} \rightarrow 0$, while also scaling $G \rightarrow 0$; i.e. violate our second condition above. Then, the theory is stringy only in the directions of $x^{0,1}$. A stringy spectrum in these directions can be removed by the ghosts, such that the whole theory has a field theoretic spectrum. In fact, we have already seen that above, when we analyzed the theory in the lightcone gauge. However, if $G_{00} \sim \alpha^{\prime} \rightarrow 0$, while some of the other components of $G_{\mu \nu}$ remain of order one, then the contribution of the energy to the dispersion relation $G^{00} p_{0}^{2} \sim \frac{1}{\alpha^{\prime}}$ is large, and the transverse oscillator modes can be excited. Therefore, in such a scaling limit the transverse oscillators do not decouple and the theory is stringy.

\section{New Noncritical String Theories}

In this section we are looking for a limit of the theory which focuses on the nearly tensionless strings for $|E| \lesssim E_{c r}(|\widetilde{E}| \lesssim 1)$. Unlike the previous section, here we keep $\alpha^{\prime}$ fixed, rather than scaling it to zero. Hence the resulting theory is a string theory and not a field theory. The limit we look for is space/time noncommutative and decouples from the bulk modes off the branes including the gravitational modes of the theory.

Since we want the open string metric $G_{\mu \nu}$ to be fixed, we should scale the closed string metric

$$
\begin{aligned}
& -g_{00}=g_{11}=g \sim \frac{1}{1-\widetilde{E}^{2}} \\
& |\widetilde{E}| \rightarrow 1,
\end{aligned}
$$

holding the other elements fixed (recall that we chose the closed string metric to be diagonal). 
Using (3.2), the noncommutativity parameter $\theta$ can be expressed in this limit as

$$
\theta=2 \pi \alpha^{\prime} G^{-1}
$$

and therefore it is finite. Hence, in this limit the theory is space/time noncommutative.

One of the surprising aspects of this theory is that the open strings, which live on the branes, cannot turn into closed strings and propagate into the bulk. The energetics argument we gave around equation (2.8) prevents such processes. We can also understand it as follows. For simplicity, let us rescale the open string metric $G_{\mu \nu}$ to $\eta_{\mu \nu}$. Then the closed string metric $g_{\mu \nu}$ is equal to $\delta_{i j}$ for $i, j \neq 0,1$ and $g_{00}=-g_{11}=-g=-1 /\left(1-\widetilde{E}^{2}\right)$. Consider a process in which open strings with energies $p_{0}^{(i)}$, spatial momenta $\left(p_{1}^{(i)}, p_{2}^{(i)}, \ldots\right)$ and masses $m^{(i)}$ attempt to escape from the branes to the bulk by turning into closed strings. The open strings dispersion relations are of the form $\left(p_{0}^{(i)}\right)^{2}=\left(p_{1}^{(i)}\right)^{2}+\left(p_{2}^{(i)}\right)^{2}+$ $\ldots+\left(m^{(i)}\right)^{2}$. As they scatter to closed strings in the bulk, the total energy and momenta in the directions of the branes are conserved but the metric used in the dispersion relation is the closed string metric. Therefore, for each closed string we have $\frac{1}{g} p_{0}^{2}=\frac{1}{g} p_{1}^{2}+p_{2}^{2}+\ldots+m^{2}$. Since $g=1 /\left(1-\widetilde{E}^{2}\right) \rightarrow \infty$ as $\widetilde{E} \rightarrow 1$, such a dispersion relation cannot be satisfied unless $p_{0}$ is infinite. We see that we need to have an infinite amount of energy in order to get off the branes - the strings are confined to the branes.

Since we scale $|E| \rightarrow E_{c r}$, the open string coupling constant $G_{s}$ vanishes. As a first attempt to have an interacting theory, we scale $N$ to infinity holding the effective 'tHooft coupling (1.10)

$$
G_{e f f}=N G_{s}=N g_{s}\left(1-\widetilde{E}^{2}\right)^{\frac{1}{2}}
$$

fixed; i.e. we scale

$$
N \sim\left(1-\widetilde{E}^{2}\right)^{-\frac{1}{2}}
$$

We conclude that our theory includes interacting open strings and the underlying spacetime is noncommutative. The open strings decouple from the closed strings, and therefore also from the gravitational sector of the theory.

It might appear surprising that we have open strings without closed strings. After all, closed strings should be found as poles in the double twist diagram. However, our limit with large $N$ suppresses the nonorientable double twist diagram, while the orientable planar diagrams survive. Our theory might include composite closed strings analogous to the QCD glueballs but these are not expected to be seen in perturbation theory and should not be confused with the closed strings which propagate off the branes. 
If the theory confines and does produce its own glueballs, their coupling constant should be proportional to $1 / N$ by topological considerations. Since we scale $N$ to infinity, the glueball coupling constant vanishes. Therefore the theory is free in terms of these variables. If a dual supergravity description exists in the strong 'tHooft coupling limit, we expect the glueballs to be described in terms of classical supergravity in some dual bulk geometry. Such a description will be useful for $G_{\text {eff }}$ large.

Another possibility, is to keep $N$ fixed and scale $g_{s}$ to infinity holding $G_{\text {eff }}$ fixed. Thus we scale

$$
g_{s} \sim\left(1-\widetilde{E}^{2}\right)^{-\frac{1}{2}}
$$

The strong coupling behavior of the theory can be analyzed using string duality. In the IIA theory we should use M-theory and in the IIB theory we should use S-duality.

Let us focus on the case of the IIB theory. We start with

$$
\begin{aligned}
& g_{s} \sim\left(1-\widetilde{E}^{2}\right)^{-\frac{1}{2}} \\
& g_{11}=-g_{00} \sim\left(1-\widetilde{E}^{2}\right)^{-1} \\
& g_{i j} \sim 1 \quad \text { for } \quad i, j \neq 0,1 .
\end{aligned}
$$

After an S-duality transformation we have

$$
\begin{aligned}
& g_{s}^{\prime} \sim\left(1-\widetilde{E}^{2}\right)^{\frac{1}{2}} \\
& g_{11}^{\prime}=-g_{00}^{\prime} \sim\left(1-\widetilde{E}^{2}\right)^{-\frac{1}{2}} \\
& g_{i j}^{\prime} \sim\left(1-\widetilde{E}^{2}\right)^{\frac{1}{2}} \quad \text { for } \quad i, j \neq 0,1 .
\end{aligned}
$$

The lightest degrees of freedom in the bulk are the fundamental strings which were originally D-strings. Their coupling constant goes to zero in the scaling limit. The original fundamental strings are D-strings after the duality transformation. Although they are strongly coupled, they are very heavy and are irrelevant for low energy processes. Of course, this last comment is consistent with the previous discussion about the decoupling of these closed strings from the modes on the branes. Since $g_{s}^{\prime} \rightarrow 0$, we do not have any closed string poles in the non-planar open string diagrams. The only poles which could be generated are poles of closed D-strings, which couple strongly. However, these are very heavy in the limit we consider and cannot be put on shell for any finite energy of the external open strings. Therefore, the energetics prevents such poles.

We also note that the nature of our D-branes changes. If we started with D3-branes, they remain D3-branes after the S-duality transformation. However, the background NS 
$B_{01}$ field becomes background RR $B_{01}$ field. So we end up with IIB theory at weak coupling with D3-branes in background RR $B_{01}$ field.

We conclude that in the low energy limit we consider, with $g_{s}$ scaling to infinity as in (4.5), the theory includes interacting open strings on the branes together with decoupled free closed strings in the bulk. The open string theory is decoupled from gravity. Again, the underlying spacetime on the D-branes is noncommutative. In this limit $N$ is kept fixed and so are the interactions of the QCD-like strings. We expect the theory to be dual to a fully interacting theory of such strings.

Our theory is reminiscent of the little string theory of [15]. In both cases we take a limit of string theory in which gravity decouples to find a theory which does not appear to be a local quantum field theory. What are the observables of the theory? In the little string theory, the S-matrix elements of the underlying string theory lead to Green's functions in the little string theory [16, 17]. Perhaps a similar construction exists for our theory but we have not investigated this.

The thermodynamics of the theory is also interesting. At weak coupling, one expects the high temperature thermodynamics of the theory to be that of a string theory with an exponentially growing density of states and a Hagedorn transition. It would be interesting to understand the behavior of the theory near the Hagedorn temperature since this theory does not contain closed strings and decouples from gravity. It would be interesting to understand the thermodynamics of the theory at large $N$ with $G_{\text {eff }}$ held fixed and large, in particular whether a Hagedorn spectrum still persists. The existence of a possible supergravity dual may help towards this direction.

In [3] the zero slope limit of D4-branes in a magnetic background was related to D4branes in a background electric field in the limit as the electric field approaches the critical value. These two limits turned out to be related from an eleven dimensional perspective in M-theory. It would be interesting to see whether this observation is relevant to our noncritical theory, as we use such a limit.

\section{Acknowledgements}

N.S. would like to thank the Theory Group at the University of Texas for hospitality during part of this work. We are greatful to R. Gopakumar, S. Minwalla and A. Strominger for a number of discussions concerning the various limits reported in this paper. We also thank M. Kleban, S. Shenker and E. Witten for useful discussions. The work of N.S. was supported in part by DOE grant \#DE-FG02-90ER40542. The work of L.S. and N.T. was supported in part by NSF grant 980115 . 


\section{References}

[1] A. Connes, M. R. Douglas, and A. Schwarz, "Noncommutative Geometry and Matrix Theory: Compactification On Tori," JHEP 9802 (1998) 003, hhep-th/9711162.

[2] M. R. Douglas and C. Hull, "D-Branes And The Noncommutative Torus," JHEP 9802 (1998) 008, hep-th/9711165.

[3] N. Seiberg and E. Witten, "String theory and noncommutative geometry," JHEP 9909 (1999) 032, hep-th/9908142.

[4] T. Yoneya, "String Theory and Space-Time Uncertainty Principle," [hep-th/0004074].

[5] N. Seiberg, L. Susskind and N. Toumbas, "The Teleological Behavior of Rigid Regge Rods" hep-th/0005015.

[6] L. Susskind, "Strings, Black Holes and Lorentz Contractions," Phys. Rev. D49 (1994) 6606; G. 't Hooft, "Horizon Operator Approach to Black Hole Quantization," gr-qc/9402037; "The scattering matrix approach for the quantum black hole, an overview," gr-qc/9607022, Int. J. Mod. Phys. A11 (1996) 4623.

[7] V. Schomerus, "D-Branes And Deformation Quantization," JHEP 9906 (1999) 030, hep-th/9903205.

[8] R. Gopakumar, J. Maldacena, S. Minwalla and A. Strominger, to appear.

[9] C. P. Burgess, "Open String Instability In Background Electric Fields," Nucl. Phys. B294 (1987) 427.

[10] C. Chu and P. Ho, "Noncommutative open string and D-brane," Nucl. Phys. B550 (1999) 151, hep-th/9812219.

[11] M. M. Sheikh-Jabbari, "Open strings in a B-field background as electric dipoles," Phys. Lett. B455 (1999) 129 hhep-th/9901080.

[12] D. Bigatti and L. Susskind, "Magnetic fields, branes and noncommutative geometry," hep-th/9908056.

[13] Z. Yin, "A note on space noncommutativity," Phys. Lett. B466 (1999) 234, hepth/9908152].

[14] S. Gukov, I. R. Klebanov and A. M. Polyakov, "Dynamics of (n,1) strings," Phys. Lett. B423 (1998) 64, hep-th/9711112.

[15] N. Seiberg, "New theories in six dimensions and matrix description of M-theory on $\mathrm{T}^{* *} 5$ and $\mathrm{T}^{* *} 5 / \mathrm{Z}(2), "$ Phys. Lett. B408 (1997) 98 hep-th/9705221.

[16] O. Aharony, M. Berkooz, D. Kutasov and N. Seiberg, "Linear dilatons, NS5-branes and holography," JHEP 9810 (1998) 004 [hep-th/9808149].

[17] S. Minwalla and N. Seiberg, "Comments on the IIA NS5-brane," JHEP 9906 (1999) 007 hep-th/9904142. 